\title{
Directly detecting isospin-violating dark matter
}

\author{
Chris Kelso, ${ }^{1}$ Jason Kumar, ${ }^{2}$ Danny Marfatia, ${ }^{2}$ and Pearl Sandick ${ }^{3}$ \\ ${ }^{1}$ Department of Physics, University of North Florida, Jacksonville, Florida 32224, USA \\ ${ }^{2}$ Department of Physics and Astronomy, University of Hawai'i, Honolulu, Hawaii 96822, USA \\ ${ }^{3}$ Department of Physics and Astronomy, University of Utah, Salt Lake City, Utah 84112, USA
}

(Received 16 November 2017; published 7 March 2018)

\begin{abstract}
We consider the prospects for multiple dark matter direct detection experiments to determine if the interactions of a dark matter candidate are isospin-violating. We focus on theoretically well-motivated examples of isospin-violating dark matter (IVDM), including models in which dark matter interactions with nuclei are mediated by a dark photon, a $Z$, or a squark. We determine that the best prospects for distinguishing IVDM from the isospin-invariant scenario arise in the cases of dark photon-or $Z$-mediated interactions, and that the ideal experimental scenario would consist of large exposure xenon- and neonbased detectors. If such models just evade current direct detection limits, then one could distinguish such models from the standard isospin-invariant case with two detectors with of order 100 ton-year exposure.
\end{abstract}

DOI: $10.1103 /$ PhysRevD.97.056004

\section{INTRODUCTION}

The most studied scenario for the direct detection of dark matter is through elastic spin-independent (SI) velocityindependent contact scattering with a variety of target nuclei. To compare the responses of various detectors, one must know the relative strength of dark matter coupling to protons $\left(f_{p}\right)$ and to neutrons $\left(f_{n}\right)$. Although it is often assumed that dark matter interactions are isospin-invariant $\left(f_{n} / f_{p}=1\right)$, it is by now well appreciated that these interactions can just as well be isospin-violating [1-5]. Isospin-violating dark matter (IVDM) has been well studied as an approach for resolving the apparent tension between the exclusion limits of some experiments (including CDMS-Ge [6,7], Edelweiss [8], XENON10 [9], XENON100 [10,11] and LUX [12]) and putative signals of other direct detection experiments (DAMA [13], CoGeNT [14,15], CRESST [16], CDMS-Si [17]). Theoretical models for this scenario have been studied in, for example, Refs. [18-24]. Our goal in this work is to consider a different set of questions: what models of IVDM are well motivated by theoretical considerations, and what types of direct detection experiments would be needed to distinguish one of these models from the more standard scenario of isospin-invariant interactions?

The difference between protons and neutrons is essentially the difference between up and down quarks, and isospin-invariant interactions generically arise in any

Published by the American Physical Society under the terms of the Creative Commons Attribution 4.0 International license. Further distribution of this work must maintain attribution to the author(s) and the published article's title, journal citation, and DOI. Funded by SCOAP ${ }^{3}$. scenario in which dark matter interactions with first generation quarks are suppressed. This situation is typical of scenarios in which the dark matter (DM) is a Majorana fermion and the theory respects minimal flavor violation (MFV); if the DM is a Majorana fermion, then SI scattering requires the quark to flip helicity, and if the theory respects MFV, then terms that flip the helicity of first generation quarks are heavily suppressed. Consequently, any theory that deviates from the assumption of Majorana fermion DM and/or MFV would naturally be expected to exhibit isospin-violating interactions with nuclei. We consider, as benchmarks, a few simple scenarios of this type.

As may be expected, one requires at least two different direct detection experiments with different target nuclei in order to distinguish a model of IVDM from the scenario of isospin-invariant interactions. We consider the scenario of an IVDM candidate which just escapes current direct detection limits from XENON1T [25] and PandaX-II [26], and we study the exposure which would be needed by two different experiments to not only discover the dark matter candidate, but determine that its interactions are isospin-violating. We will see that for the benchmark models presented here, a high- $A$ target (such as xenon, argon or germanium) and a low- $A$ target (such as neon or helium) are required. Even so, we will show that if DM-SM interactions are mediated by QCD-charged scalars, then although dark matter interactions could be isospinviolating, it would nevertheless be very difficult to distinguish this model from an isospin-invariant scenario. On the other hand, if DM-SM interactions are dark photon-or $Z$-mediated, then it would be possible to exclude isospin invariance with reasonable exposures of next generation direct detection experiments. 
The plan of this work is as follows. In Sec. II, we describe a variety of theoretically motivated models of isospin-violating dark matter. In Sec. III, we describe the analytical framework for distinguishing these models using data from multiple direct detection experiments. We present our results in Sec. IV and conclude in Sec. V.

\section{THEORETICALLY MOTIVATED IVDM MODELS}

The typical scenario of isospin-invariant DM-nuclei interactions arises if SI-interactions between DM and first generation quarks are suppressed, since it is only the first generation quark content of the nucleon which distinguishes protons from neutrons. This scenario is most often realized in models in which the dark matter is a Majorana fermion and the theory respects minimal flavor violation. This is often the case, for example, in the Minimal Supersymmetric Standard Model (MSSM).

If dark matter is a fermion, velocity-unsuppressed SI elastic scattering only arises from matrix element terms coupling the scalar or vector DM current to the same quark current (see [27], for example). But if dark matter is a Majorana fermion, then the vector current necessarily vanishes, and SI scattering can only arise from a coupling of a scalar DM current to a scalar quark current. But a coupling to the scalar quark current necessarily flips the quark helicity, which violates SM flavor symmetries. Under the assumption of MFV, any such violation of SM flavor symmetries must be proportional to the Yukawa couplings, implying that any coupling to the scalar current of first generation quarks is heavily suppressed. Thus, the assumptions of Majorana fermion dark matter and MFV are sufficient to suppress isospin-violating DM interactions, regardless of the microscopic details of the model. Indeed, both of these assumptions are realized by the most studied WIMP candidate, the lightest neutralino of the constrained MSSM (CMSSM). But by the same token, a deviation from either of these assumptions will generically lead to IVDM (unless dark matter couples to up and down quarks in the same way).

We consider three benchmark examples for the interaction of a generic DM candidate with SM quarks.

(a) Dark Photon Mediation: The dark matter is a Dirac fermion which interacts through a massive dark photon [28-31] that kinetically mixes with the SM photon.

(b) Z Mediation: The dark matter is a Dirac fermion which interacts through $Z$ exchange.

(c) Squark Mediation: The dark matter is a binolike lightest neutralino of the MSSM, which interacts with nucleons through squark exchange [32], but flavor violation is not minimal.

\section{A. Dark photon-mediated interactions}

In this scenario, dark matter is a Dirac fermion $(X)$, and the DM vector current couples to a massive dark photon
$\left(A^{\prime}\right)$ which kinetically mixes with the photon [33]. With a suitable field redefinition, one can diagonalize the kinetic terms of the $\left(A, A^{\prime}\right)$ Lagrangian, inducing a small coupling of charged SM particles to $A^{\prime}$. At low energies, the effective interaction can be expressed as a contact operator of the form $\left(1 / \Lambda^{2}\right)\left(\bar{X} \gamma^{\mu} X\right)\left(\bar{q} \gamma_{\mu} q\right)$, which generates SI scattering. But necessarily, couplings of SM particles to the dark photon mediator are proportional to the particle's electromagnetic charge. We thus find that the DM-neutron coupling vanishes, and

$$
\left(\frac{f_{n}}{f_{p}}\right)_{A^{\prime}}=0
$$

\section{B. Z-mediated interactions}

In this scenario, dark matter is again a Dirac fermion $(X)$ which couples to the $Z$. In this case, the relative couplings of dark matter to neutrons and protons are entirely determined by the coupling of the $Z$ to nucleons, and we find

$$
\left(\frac{f_{n}}{f_{p}}\right)_{Z}=\frac{-1 / 2}{1 / 2-2 \sin ^{2} \theta_{W}} \approx-12.5 .
$$

This scenario is the counterpoint to the dark photon-mediated scenario; whereas dark photon-mediated interactions lead to vanishing DM-neutron couplings, $Z$-mediated interactions lead to heavily suppressed DM-proton couplings.

\section{Squark-mediated interactions}

In this scenario, the DM candidate is the lightest neutralino of the MSSM, which is taken to be binolike. Velocity-independent SI scattering can then be mediated by $u-/ s$-channel squark exchange, but the scattering matrix element is necessarily proportional to the left-right squark mixing angle. If the theory does not respect MFV, then the light-flavored squark mixing angles need not be small, implying that the SI-scattering cross section can be significant even though the DM candidate is a Majorana fermion. This scenario has also been considered in $[34,35]$.

We consider the case where one squark ( $\tilde{q})$ is significantly lighter than the others, and thus dominates DMnucleon scattering processes. Of course, gauge invariance requires that both up-type and down-type quarks be present in the spectrum, so one cannot strictly decouple one member of an $S U(2)_{L}$ doublet. We consider the limit of one light squark only for the purpose of identifying a benchmark for the largest deviation from isospin-invariant interactions that could be obtained in the scenario of squark-mediated interactions. This benchmark will tend to be realized in scenarios in which the mass splitting between the dark matter and the lightest squark is smaller than the mass splitting between different squarks.

After integrating out the light squark, the DM-quark interaction relevant for SI scattering can be expressed in 
terms of the contact operator $\left(1 / \Lambda^{2}\right)(\bar{X} X)(\bar{q} q)$. In this case, $\mathrm{DM}$ has non-negligible coupling only to one quark flavor, and $f_{n} / f_{p}$ is entirely determined by the quark content of the nucleons. We find

$$
\left(\frac{f_{n}}{f_{p}}\right)_{\tilde{q}}=\frac{B_{q}^{n}}{B_{q}^{p}}
$$

where $B_{q}^{n, p}$ are the nucleon form factors associated with the scalar current, and $q$ is the flavor of the light squark.

If the light squark is $\tilde{s}$, then $f_{n} / f_{p} \sim 1$, as the strangeness content of the neutron and proton are nearly identical. On the other hand, if the light squark is either $\tilde{u}$ or $\tilde{d}$, then the relevant nucleon form factors have considerable uncertainty, related to the strangeness content of the nucleon. The larger the strangeness content of the nucleon, the closer $f_{n} / f_{p}$ will be to one. But recent lattice QCD results and more modern chiral perturbation theory calculations suggest that the strangeness content of the nucleon might be very small. For the purposes of this benchmark, we consider the case in which the strangeness content of the nucleon is taken to be as small as is reasonably possible, namely, the value it would assume in the limit where the strange quark can be treated as a heavy quark. This case was considered in [34], and it was found that, in this limit, reasonable values for the remaining nucleon form factors are given by

$$
\begin{aligned}
B_{u}^{p} & =B_{d}^{n} \sim 9.95, \\
B_{u}^{n} & =B_{d}^{p} \sim 6.6 .
\end{aligned}
$$

With these values,

$$
\begin{aligned}
& \left(\frac{f_{n}}{f_{p}}\right)_{\tilde{u}}=\frac{B_{u}^{n}}{B_{u}^{p}} \sim 0.67, \\
& \left(\frac{f_{n}}{f_{p}}\right)_{\tilde{d}}=\frac{B_{d}^{n}}{B_{d}^{p}} \sim 1.49,
\end{aligned}
$$

for the benchmark cases of one light up-type squark and one light down-type squark, respectively.

In Table I, we summarize the benchmark scenarios considered in this paper. We present both $f_{n} / f_{p}$ and its inverse, which will be useful in the following analysis.

TABLE I. Table of $f_{n} / f_{p}$ and its inverse for the four benchmark models discussed in the text.

\begin{tabular}{lcc}
\hline \hline Model & $f_{n} / f_{p}$ & $f_{p} / f_{n}$ \\
\hline$A^{\prime}$-mediated & 0 & $\infty$ \\
$Z$-mediated & -12.5 & -0.08 \\
$\tilde{u}$-mediated & 0.67 & 1.49 \\
$\tilde{d}$-mediated & 1.49 & 0.67 \\
\hline \hline
\end{tabular}

\section{ANALYTICAL FRAMEWORK FOR DISTINGUISHING IVDM MODELS}

The elastic scattering event rate at a direct detection experiment with a particular target isotope is given by

$$
\frac{d R_{(Z, A)}}{d E_{R}}=n_{T} \frac{\rho_{X}}{m_{X}} \epsilon_{Z}\left(E_{R}\right) \int_{v_{\min }\left(E_{R}\right)}^{v_{\max }} d^{3} v f(v) v \frac{d \sigma_{\mathrm{SI}}^{Z, A}}{d E_{R}},
$$

where $v_{\min }\left(E_{R}\right)=\left(m_{A} E_{R}\right)^{1 / 2} / \sqrt{2} \mu_{A}$ is the minimum dark matter velocity needed to produce an elastic scatter with recoil energy $E_{R}, v_{\max }$ is the Galactic escape velocity in the Earth's frame, $n_{T}$ is the number of target nuclei of mass $m_{A}$, $\mu_{A}=m_{X} m_{A} /\left(m_{A}+m_{X}\right)$ is the dark matter-nucleus reduced mass, and $f(v)$ is the dark matter velocity distribution. $\epsilon_{Z}\left(E_{R}\right)$ is the efficiency of the detector to detect nuclear recoils of energy $E_{R}$; we assume that this efficiency depends on the detector type, but is largely independent of the particular isotope. We take the local density of dark matter to be $\rho_{X} \sim 0.3 \mathrm{GeV} / \mathrm{cm}^{3}$. The total event rate is then the sum of the scattering event rates for each isotope in the detector.

If dark matter-nucleon scattering is mediated by an isospin-violating velocity-independent contact interaction, then the differential scattering cross section can be expressed as

$\frac{d \sigma_{\mathrm{SI}}^{Z, A}}{d E_{R}}=\frac{m_{A}}{2 \mu_{p}^{2} v^{2}} \sigma_{\mathrm{SI}}^{p}\left[Z F_{A}^{p}\left(E_{R}\right)+\left(\frac{f_{n}}{f_{p}}\right)(A-Z) F_{A}^{n}\left(E_{R}\right)\right]^{2}$,

where $\mu_{p}$ is the dark matter-proton reduced mass, $\sigma_{\mathrm{SI}}^{p}$ is the dark matter-proton scattering cross section, and the $F_{A}^{n, p}\left(E_{R}\right)$ are the Helm nuclear form factors appropriate for velocity-independent SI scattering.

The total scattering event spectrum is then

$$
\begin{aligned}
\frac{d N}{d E_{R}}= & \sum_{Z, A} \frac{M_{Z, A} T \sigma_{\mathrm{SI}}^{p}}{m_{X}} \frac{\rho_{X}}{2 m_{p}^{2}} \\
& \times\left[Z F_{A}^{p}\left(E_{R}\right)+\left(\frac{f_{n}}{f_{p}}\right)(A-Z) F_{A}^{n}\left(E_{R}\right)\right]^{2} \\
& \times \epsilon_{Z}\left(E_{R}\right)\left[\int_{v_{\min }\left(E_{R}\right)}^{v_{\max }} d^{3} v \frac{f(v)}{v}\right],
\end{aligned}
$$

where $T$ is the live time, $M_{Z, A}$ is the total detector mass of the given isotope, and the sum is over all isotopes in the given detector. We have assumed $m_{X} \gg m_{p}$. Note that the only dependence of the event spectrum on $m_{X}$ arises from the overall $m_{X}^{-1}$ scaling, and from the dependence of $v_{\min }$ on $m_{X}$, via its dependence on $\mu_{A}$.

Equation (8) encapsulates everything we need to estimate the ability of direct detection experiments to distinguish a model of IVDM from isospin-invariant dark matter. Given model parameters $\left(\sigma_{\mathrm{SI}}^{p}, m_{X}, f_{n} / f_{p}\right)$ and detector parameters 
$\left(M_{Z, A}, T\right)$, Eq. (8) determines the number of elastic scattering events in each recoil energy bin, for any type of detector.

For this analysis, we focus on the scenario in which the dark matter candidate evades current direct detection limits but the neutrino background event rate is negligible compared to the DM scattering event rate. Similarly, we assume that $M_{Z, A}$ represent the total detector isotope mass within a fiducial volume chosen so that the detector background event rate (from radiogenic sources, etc.) is negligible compared to the DM scattering event rate. This is a reasonable assumption, because the DM scattering event rate scales with detector exposure, while the detector background event rate for a liquid noble time projection chamber will not, due to self-shielding. Under these assumptions, there is effectively no background, and the event spectra at any two detectors are entirely determined by Eq. (8). Then, determination of the exposures needed for two direct detection experiments to distinguish a given IVDM model from the $f_{n} / f_{p}=1$ case is a purely statistical question.

Although we present a detailed numerical analysis in the next section, one finds that the statistical analysis greatly simplifies, and can indeed be performed analytically, in the limit where $m_{X} \gg m_{A}$ for all relevant target nuclei. In this limit, $\mu_{A} \sim m_{A}$ and $v_{\min }\left(E_{R}\right) \sim\left(E_{R} / 2 m_{A}\right)^{1 / 2}$. The scattering event spectrum thus depends on $\sigma_{\mathrm{SI}}^{p}$ and $m_{X}$ only through the overall factor $\left(\sigma_{\mathrm{SI}}^{p} / m_{X}\right)$. In order to facilitate an analytical analysis, we make two additional simplifying approximations (these approximations are not made in the following numerical analysis). First, we ignore the small variation of $v_{\min }\left(E_{R}\right)$ with isotope mass for fixed $E_{R}$. Second, we ignore the variation of the Helm form factor between different isotopes, and between protons and neutrons $\left(F_{A}^{n}\left(E_{R}\right)=F_{A}^{p}\left(E_{R}\right)=F_{A}\left(E_{R}\right)\right)$. Under these approximations, the scattering event spectrum simplifies, yielding

$$
\begin{aligned}
\frac{d N}{d E_{R}}= & \frac{M_{\mathrm{total}} T \sigma_{\mathrm{SI}}^{p}}{m_{X}} \frac{\rho_{X}}{2 m_{p}^{2}}\left[\sum_{i} \eta_{i}\left[Z+\left(f_{n} / f_{p}\right)\left(A_{i}-Z\right)\right]^{2}\right] \\
& \times G\left(E_{R}\right),
\end{aligned}
$$

where

$$
G\left(E_{R}\right) \equiv F_{A}^{2}\left(E_{R}\right) \epsilon_{Z}\left(E_{R}\right)\left[\int_{\left(E_{R} / 2 m_{A}\right)^{1 / 2}}^{v_{\max }} d^{3} v \frac{f(v)}{v}\right],
$$

and the summation over $i$ is over target isotopes $\left(Z, A_{i}\right)$ with abundances $\eta_{i}$. $M_{\text {total }}$ is the total detector target mass. For any detector, $G\left(E_{R}\right)$ depends on the structure of the nuclei and the dark matter velocity distribution, but is independent of the nature of the dark matter particle.

Direct detection experiments report a normalized-tonucleon cross section $\sigma_{Z}^{N}$, which is defined as

$$
\sigma_{Z}^{N} \equiv \sigma_{\mathrm{SI}}^{p} \frac{\sum_{i} \eta_{i}\left[Z+\left(f_{n} / f_{p}\right)\left(A_{i}-Z\right)\right]^{2}}{\sum_{i} \eta_{i} A_{i}^{2}} \equiv \frac{\sigma_{\mathrm{SI}}^{p}}{F_{Z}}
$$

Note that this definition of $F_{Z}$ differs from that in [4] only by the assumption $\mu_{A_{i}} \sim \mu_{A}$. In terms of this quantity, the scattering event spectrum can be expressed as

$$
\frac{d N}{d E_{R}}=\frac{\sigma_{Z}^{N}}{m_{X}} \frac{\rho_{X} M_{\text {total }} T}{2 m_{p}^{2}}\left(\sum_{i} \eta_{i} A_{i}^{2}\right) G\left(E_{R}\right) .
$$

Given fixed assumptions about the dark matter velocity distribution and the nuclear form factors, a signal at a direct detection experiment really provides a measurement of $X_{Z} \equiv \sigma_{Z}^{N} / m_{X}$, in the limit $m_{X} \gg m_{A}$. We are interested in the quantity [4]

$$
R\left[Z_{1}, Z_{2}\right]\left(f_{n} / f_{p}\right) \equiv \frac{\sigma_{Z_{1}}^{N}}{\sigma_{Z_{2}}^{N}}=\frac{F_{Z_{2}}}{F_{Z_{1}}}=\frac{X_{Z_{1}}}{X_{Z_{2}}},
$$

because for a given pair of nuclei with $Z_{1}$ and $Z_{2}$ protons, it is entirely determined by $f_{n} / f_{p}$.

We may express the fractional uncertainty in $R\left[Z_{1}, Z_{2}\right]$ $\left(f_{n} / f_{p}\right)$ as

$$
\begin{aligned}
\frac{\delta R\left[Z_{1}, Z_{2}\right]\left(f_{n} / f_{p}\right)}{R\left[Z_{1}, Z_{2}\right]\left(f_{n} / f_{p}\right)} & =\sqrt{\left(\frac{\delta X_{Z_{1}}}{X_{Z_{1}}}\right)^{2}+\left(\frac{\delta X_{Z_{2}}}{X_{Z_{2}}}\right)^{2}} \\
& =\sqrt{\frac{1}{N_{Z_{1}}}+\frac{1}{N_{Z_{2}}}}
\end{aligned}
$$

where $N_{Z_{i}}=\int d E_{R}\left(d N_{Z_{i}} / d E_{R}\right)$ is the total number of DM scattering events in the detector made of nuclei with $Z_{i}$ protons. One only needs to determine the exposures needed to ensure that the fractional uncertainty in the measurement of $R\left[Z_{1}, Z_{2}\right]\left(f_{n} / f_{p}\right)$ is small enough that the result can be statistically distinguished from $R\left[Z_{1}, Z_{2}\right]\left(f_{n} / f_{p}=1\right)=1$. It is worth noting that even if one obtains a very large exposure with one experiment, there is still a minimum exposure of a second experiment required to obtain any given precision in the measurement of $R$. In particular, one can obtain a fixed precision in the measurement of $R$ with two experiments whose exposures are each large enough to yield $N=N_{Z_{1}}=N_{Z_{2}}=2(\delta R / R)^{-2}$ events in each experiment. But even if one obtains an arbitrarily large exposure with one detector, the exposure needed from the second detector to obtain the same precision is only reduced by a factor of 2 .

Since backgrounds are negligible in our scenario, a direct detection experiment will find initial evidence for dark matter interactions when it observes $\sim 2-3$ scattering events. Thus, to obtain $\sim 2 \sigma$ evidence of a $10 \%$ deviation of $R\left[Z_{1}, Z_{2}\right]\left(f_{n} / f_{p}\right)$ from 1 would require each detector to have $N_{Z_{i}} \gtrsim 800$ events, which amounts to a exposure 


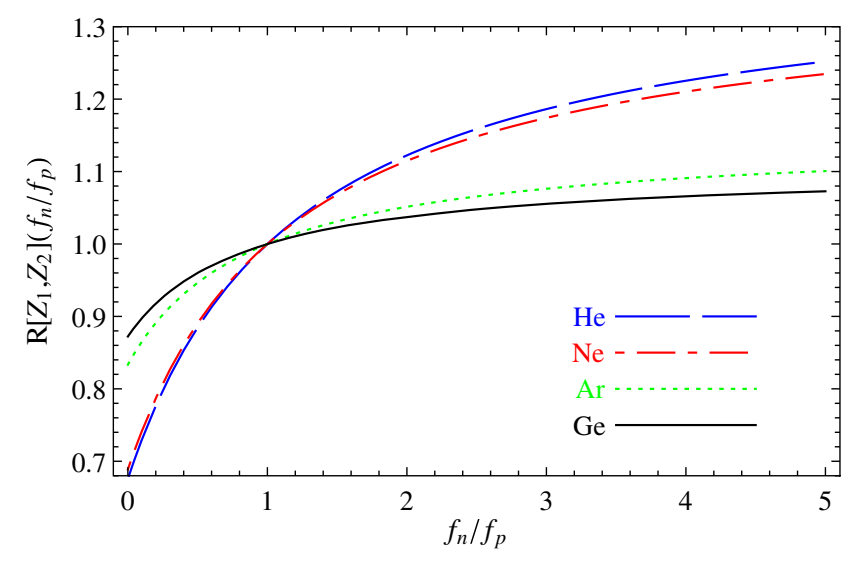

FIG. 1. $R\left[Z_{1}, Z_{2}\right]$, as a function of $f_{n} / f_{p}$, for $Z_{1}=\mathrm{Xe}$ and $Z_{2}=\mathrm{He}, \mathrm{Ne}$, Ar or Ge.

300-400 times longer than that needed to first see evidence of dark matter interactions. If evidence of dark matter interactions appeared in the initial short-time run of a direct detection experiment, then the full physics run of a next generation detector (with the same target material) may provide the increase in exposure needed to resolve $R\left[Z_{1}, Z_{2}\right]\left(f_{n} / f_{p}\right)$ at the $10 \%$ level. But if one needed to resolve $R\left[Z_{1}, Z_{2}\right]\left(f_{n} / f_{p}\right)$ at the $1 \%$ level, an additional $\times 100$ increase in exposure would be needed.

For simplicity, we assume that one of the two relevant direct detection experiments will be xenon-based, while the other is helium-, neon-, argon- or germanium-based. In Fig. 1, we present our numerical results for $R\left[\mathrm{Xe}, Z_{2}\right]$ $\left(f_{n} / f_{p}\right)$. All curves cross at $\left(f_{n} / f_{p}=1.0, R\left[\mathrm{Xe}, Z_{2}\right]=\right.$ $1.0)$, as expected. In Table II, we list the relevant values of $R\left[\mathrm{Xe}, Z_{2}\right]\left(f_{n} / f_{p}\right)$ for each of our benchmark scenarios and for each of the relevant detector targets. Note that since none of the benchmarks present a cancellation between proton and neutron couplings, the differences between the responses of different isotopes for each element are negligible. For the purpose of distinguishing $R$ from 1 , it is clear that the most promising choices for the second target are low- $Z$ materials such as neon. Argon and germanium are both high- $Z$ materials, like xenon, for which there are more neutrons than protons. For all of the benchmark scenarios, a significantly higher precision in the determination of $R$ is needed if both targets are high- $Z$ than is needed if one target is low- $Z$.

TABLE II. Values of $R\left[\mathrm{Xe}, Z_{2}\right]\left(f_{n} / f_{p}\right)$ for the four benchmark models and $Z_{2}=\mathrm{He}, \mathrm{Ne}, \mathrm{Ar}$ or Ge.

\begin{tabular}{lcccc}
\hline \hline & \multicolumn{4}{c}{ Second Target } \\
\cline { 2 - 5 } Model & $\mathrm{He}$ & $\mathrm{Ne}$ & $\mathrm{Ar}$ & $\mathrm{Ge}$ \\
\hline$A^{\prime}$-mediated & 0.68 & 0.69 & 0.83 & 0.87 \\
$Z$-mediated & 1.46 & 1.43 & 1.17 & 1.12 \\
$\tilde{u}$-mediated & 0.93 & 0.93 & 0.97 & 0.98 \\
$\tilde{d}$-mediated & 1.07 & 1.07 & 1.03 & 1.02 \\
\hline \hline
\end{tabular}

We also see that for the case of first generation squarkmediated interactions, $R$ must be determined at the few percent level, regardless of the choice of the second detector. Such a determination would thus be challenging even for detectors at the generation beyond the experiments which first provided evidence for dark matter. For $Z$ - or dark photon-mediated interactions, however, prospects are much more promising, since $R$ need only be measured at the level of a few tens of percent.

\section{RESULTS}

In this section we present the results of a full numerical analysis of the ability of two future dark matter direct detection experiments to distinguish typical scenarios of IVDM from the default hypothesis of $f_{n} / f_{p}=1$, after marginalizing over $m_{X}$ and $\sigma_{\mathrm{SI}}^{p}$. The case in which $f_{n} / f_{p} \sim-1$, with cancellations between the responses of protons and neutrons, has been well studied in the literature (see, for example, [5,36]). We instead focus on our benchmarks, for which there are no large cancellations. We have seen from Fig. 1 that the best prospects for distinguishing IVDM from isospin-invariant dark matter then arise when one detector uses a high- $Z$ target, while the other uses a low$Z$ target. We thus assume that the two available detectors use xenon and neon as targets.

We assume that, for any choice of $f_{n} / f_{p}$, the true value of $\sigma_{\mathrm{SI}}^{p}$ is chosen so that the model evades current limits from direct detection experiments. But we also assume that $\sigma_{\mathrm{SI}}^{p}$ is large enough that the dark matter scattering event rate at either xenon-based or neon-based direct detection experiments is larger than the neutrino background rate. This latter assumption ensures that the background-free approximation is still valid.

Our statistical analysis depends only on the number of events observed at each experiment, which is proportional to (exposure) $\times \sigma_{\mathrm{SI}}^{p}$. We therefore define an effective exposure which is given by

effective exposure $\equiv($ exposure $) \times \frac{\sigma_{\mathrm{SI}}^{p}}{\sigma_{\mathrm{SI}}^{p, \text { Xe-limit }}\left(m_{X}\right)}$,

where $\sigma_{\mathrm{SI}}^{p \text {,Xe-limit }}\left(m_{X}\right)$ is the current $90 \%$ C.L. bound on $\sigma_{\mathrm{SI}}^{p}$ from xenon-based experiments. ${ }^{1}$ We present our analysis in terms of effective exposures, which encode all dependence on $\sigma_{\mathrm{SI}}^{p}$.

For simulated data corresponding to a set of true values of $\sigma_{\mathrm{SI}}^{p}, m_{X}$ and $f_{n} / f_{p}$ (or $f_{p} / f_{n}$ ), we find the best fit mass and cross section assuming $f_{n} / f_{p}=1$. We then determine the confidence level at which the best fit point can be

\footnotetext{
${ }^{1}$ For squark-mediated models, the LHC places tight lower bounds on the squark mass. But, for example, a model with $m_{X} \sim 1000 \mathrm{GeV}$, a squark-bino mass splitting of $\mathcal{O}(15 \mathrm{GeV})$ and a left-right squark mixing angle of $\mathcal{O}\left(10^{-3}\right)$, escapes LHC constraints with $\sigma_{\mathrm{SI}}^{p}$ at the current XENON1T limit [35].
} 
excluded by comparing its $\chi^{2}$ to that of the true model $\left(\chi^{2}=0\right)$ using a $\Delta \chi^{2}$ test with two free parameters. In Fig. 2, we plot exclusion contours of the $f_{n} / f_{p}=1$ scenario, as a function of the true value of $f_{n} / f_{p}$ (left panels) or $f_{p} / f_{n}$, (right panels), and the effective exposure of a future neon-based direct detection experiment with the same efficiency and recoil energy window as microCLEAN [37]. We take the true values of the dark matter mass to be $m_{X}=10 \mathrm{GeV}$ (top panels), $100 \mathrm{GeV}$ (middle panels) and $1000 \mathrm{GeV}$ (bottom panels). We have assumed that we also have data from a future xenon-based experiment with a 100 ton-year effective exposure, with the same efficiency and recoil energy window as XENON1T. Given this effective exposure at a xenon-based experiment, one would expect $\sim 2200$ events if $m_{X} \gtrsim 100 \mathrm{GeV} .^{2}$ For this exposure, $R[\mathrm{Xe}, \mathrm{Ne}]$ can be resolved within $\mathcal{O}(2-3 \%)$ with a sufficiently long exposure of a neon-based experiment.

The exclusion contours of the $f_{n} / f_{p}=1$ hypothesis are at $2 \sigma$ (purple short/long-dashed), $3 \sigma$ (green short-dashed), $4 \sigma$ (black solid) and $5 \sigma$ (blue long-dashed) confidence. The vertical grey dashed lines in each panel correspond to the values of $f_{n} / f_{p}$ (left panels) or $f_{p} / f_{n}$ (right panels) expected for our benchmark models. In all panels, the solid red contour is the neon effective exposure necessary for an expected 2.3 signal events; if no events are observed, such a model would be excluded at $90 \%$ C.L. Unless $f_{n} / f_{p} \approx-1$, an observation of DM-nucleon SI elastic scattering will be achieved with a neon experiment with a modest effective exposure of at most a few ton-years. Typically, a far larger effective exposure is necessary to exclude the $f_{n} / f_{p}=1.0$ scenario.

Focusing first on the left column of panels, for $f_{n} / f_{p} \approx-0.7$, a very small neon effective exposure $\left(\lesssim 0.02\right.$ ton-year) would be sufficient to exclude $f_{n} / f_{p}=$ 1 to high significance. This is due to the fact that a xenon experiment is largely insensitive to $f_{n} / f_{p} \approx-0.7$, where cancellations result in a very large value for $F_{\mathrm{Xe}}$. If a future $\sim 100$ ton-year effective exposure xenon experiment sees no signal, then any signal in a neon detector would yield high confidence that $f_{n} / f_{p} \neq 1$. Similarly, for $f_{n} / f_{p} \approx-0.98$, cancellations result in an insensitivity of neon-based experiments. In this case, the absence of events in a neon-based experiment, combined with a large number of events in a xenon-based experiment, would be sufficient to exclude $f_{n} / f_{p}=1$. Between these values, for $f_{n} / f_{p} \approx-0.83$, each experiment suffers approximately the same suppression of sensitivity, and we find $F_{\mathrm{Xe}} \sim F_{\mathrm{Ne}}, R[\mathrm{Xe}, \mathrm{Ne}] \sim 1$ [36]. The data can thus be very well fit by the $f_{n} / f_{p}=1$ model, simply by rescaling $\sigma_{\mathrm{SI}}^{p}$. As a result, if $f_{n} / f_{p} \approx-0.83$, it will be

\footnotetext{
${ }^{2}$ For $m_{X}=10 \mathrm{GeV}$, the number of events would be about a factor of 4 smaller. This dependence on mass arises because the current XENON1T bound is not based on a cut-and-count analysis. At small mass, the current sensitivity is not directly connected to the number of events in the recoil energy window.
}

effectively impossible to distinguish this from $f_{n} / f_{p}=1$ with even a several hundred ton-year effective exposure neon experiment. However, for different target nuclei, these cancellations occur for different values of $f_{n} / f_{p}$, so it may be possible to address this particular value of $f_{n} / f_{p}$ with a germanium or argon experiment; see the Appendix.

For larger values of $f_{n} / f_{p}$, we see that for $f_{n} / f_{p} \gtrsim-0.7$ the exclusion contours run to very large values for the neon effective exposure-a very large neon effective exposure would be necessary to see any discrepancy with $f_{n} / f_{p}=1$. In the left panels of Fig. 2, the vertical grey dashed lines correspond to the values of $f_{n} / f_{p}$ expected for $A^{\prime}$ mediation, $\tilde{u}$ mediation and $\tilde{d}$ mediation, from left to right. While the squark-mediation scenarios will not be probed with realistic future dark matter direct detection experiments, we find that, in the case of $A^{\prime}$-mediated interactions, a $2 \sigma$ discrepancy with $f_{n} / f_{p}=1$ would be found with $\sim 100$ ton-year effective exposure of a xenon experiment and $\lesssim 50$ ton-year effective exposure of a neon experiment. This discrepancy could rise to $3 \sigma$ with approximately 100 ton-years of data from each of the two experiments. We note that for modestly larger values of $f_{n} / f_{p}$, a signal would emerge in a neon detector with relatively low exposure, typically only a few ton-years, but it is quite challenging, even for the case of $Z_{1}=\mathrm{Xe}$ and $Z_{2}=\mathrm{Ne}$, a high mass and a low-mass target, to distinguish IVDM from $f_{n} / f_{p}=1$.

Next, we turn to the right column of panels of Fig. 2, which are similar to the left panels but as a function of $f_{p} / f_{n}$. Thus, $\left|f_{p} / f_{n}\right| \approx 0$ corresponds to very large $\left|f_{n} / f_{p}\right|$. Again, we see that for $f_{n} \approx-f_{p}$, the neon experiment will see a dramatic decrease in sensitivity to DM scattering such that several tens of ton-years of effective exposure may be necessary to observe even a few scattering events. The vertical grey dashed lines correspond to the values of $f_{p} / f_{n}$ expected for $Z$ mediation, $\tilde{d}$ mediation and $\tilde{u}$ mediation, from left to right. The right panels reinforce that the squark-mediated models will not be accessible, but here we see that a $2 \sigma$ discrepancy with $f_{n} / f_{p}=1$ would be discovered with $\sim 100$ ton-year effective exposure of a xenon experiment and approximately 50 ton-year effective exposure of a neon experiment if $f_{p} / f_{n} \approx-0.08$, as would be expected if Dirac fermion DM scatters with quarks via $Z$ exchange.

We can apply the results of our analytic study of the heavy dark matter limit if $m_{X} \gg m_{\mathrm{Xe}}$. Since $R[\mathrm{Xe}, \mathrm{Ne}]$ $\left(f_{n} / f_{p}=0\right) \sim 0.7$, the uncertainty in the measurement of $R$ is largely determined by the number of expected events at the neon-based detector. In the heavy dark matter limit, one then requires $N_{\mathrm{Ne}} \sim(0.15)^{-2} \sim 44$ events at a neon-based detector in order to exclude the $f_{n} / f_{p}=1$ scenario at $2 \sigma$ confidence, implying that the necessary effective exposure is about a factor of 20 larger than that needed to obtain 2.3 expected signal events. We see that this expectation is borne out by the results of Fig. 2 for $m_{X}=1000 \mathrm{GeV}$. 

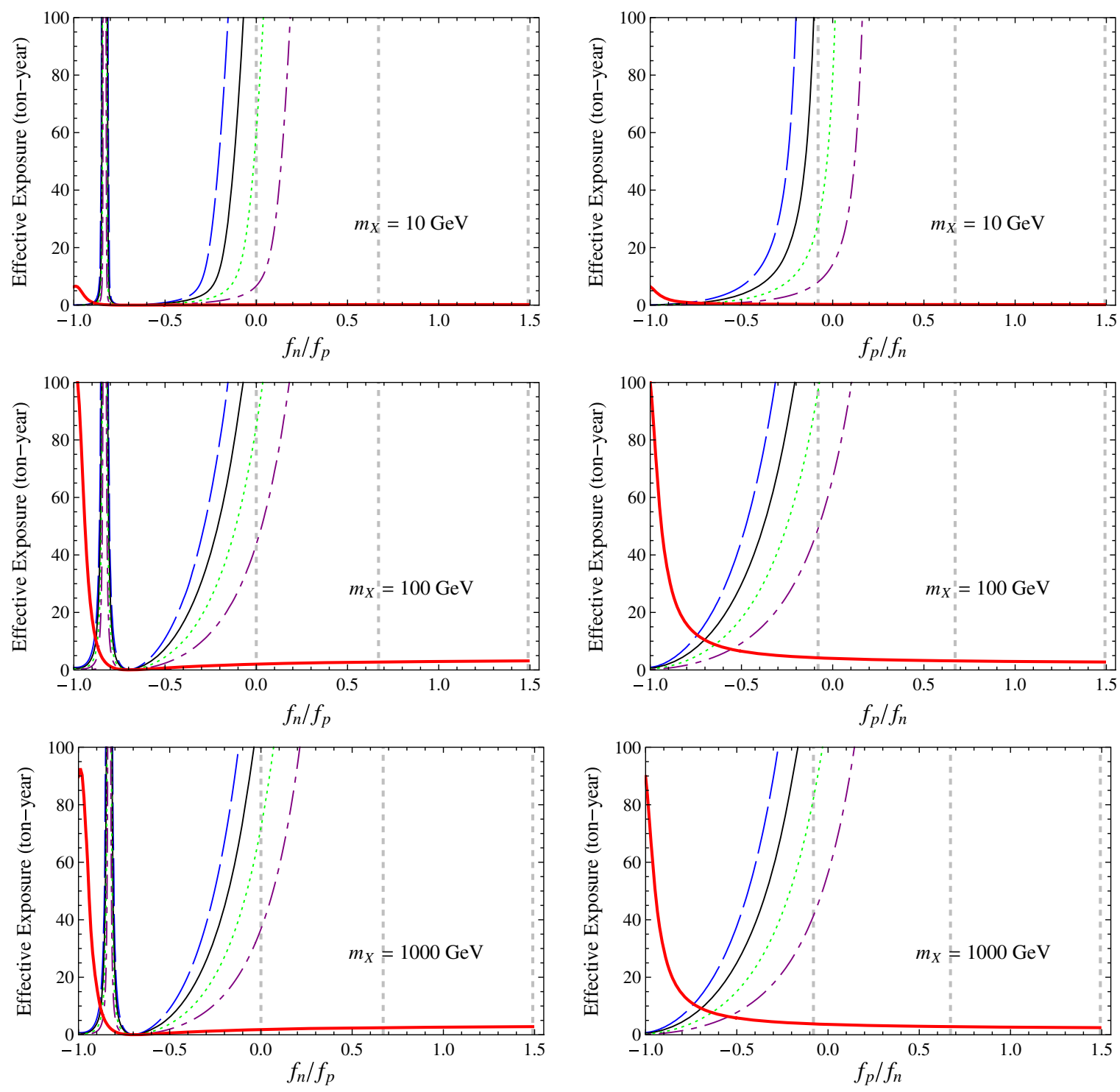

FIG. 2. Exclusion contours of the $f_{n} / f_{p}=1$ scenario, in terms of the true value of $f_{n} / f_{p}$ (left panels) or $f_{p} / f_{n}$ (right panels) and the effective exposure of a neon-based experiment, assuming a 100 ton-year effective exposure at a xenon-based experiment. The exclusion contours of the $f_{n} / f_{p}=1$ hypothesis are at $2 \sigma$ (purple short/long-dashed), $3 \sigma$ (green short-dashed), $4 \sigma$ (black solid) and $5 \sigma$ (blue longdashed) confidence. The dashed grey lines correspond, from left to right, to the benchmark cases of $A^{\prime}$ mediation, $\tilde{u}$ mediation, $\tilde{d}$ mediation (left panels), and $Z$ mediation, $\tilde{d}$ mediation, $\tilde{u}$ mediation (right panels). The red line is the effective exposure needed to have 2.3 expected events at a neon-based experiment.

For any $m_{X} \gg m_{\mathrm{Xe}}$, the exclusion contours are nearly the same as in the bottom panels of Fig. 2. This is because, for any detector, the event rate is proportional to $\sigma_{\mathrm{SI}}^{p} / m_{X}$ for $m_{X} \gg m_{A}$. Thus, if $m_{X} \gg m_{\mathrm{Xe}}$, then $\sigma_{\mathrm{SI}}^{p \text {,Xe-limit }} \propto m_{X}$, which implies that the effective exposure of any detector is proportional the expected number of events, independent of the model parameters. The effective exposure of a neonbased experiment needed to exclude the isospin-invariant scenario is then independent of $m_{X}$ if $m_{X} \gg m_{\mathrm{Xe}}$.

But the effective exposure needed for a neon-based detector to have 2.3 expected events is significantly smaller for $m_{X} \sim 10 \mathrm{GeV}$. For such a small dark matter mass, a large fraction of scattering events at a xenonbased detector will fall below the recoil energy threshold, while a much smaller fraction will fall below the threshold we have assumed for a neon-based experiment. The reduction in the event rate at a xenon-based detector relative to a neon-based detector results in a reduction in the effective exposure needed to obtain a fixed number of events at the neon-based detector. The shape of the exclusion contours is also significantly different at small $m_{X}$ because marginalization over the mass has a nontrivial effect on the shape of the recoil energy spectrum. 


\section{CONCLUSIONS}

In this work, we have revisited isospin-violating dark matter with the goal of identifying theoretically wellmotivated values for the relative coupling to neutrons and protons $\left(f_{n} / f_{p}\right)$, and determining the prospects for distinguishing such a model from the standard scenario of isospin-invariant interactions using two different direct detection experiments. As has been previously noted in the literature, the most dramatic effect on direct detection sensitivity occurs when $f_{n} / f_{p} \sim-\mathcal{O}(1)$. In this case, cancellations between the response of protons and neutrons can drastically suppress the event rate in one detector, providing a telltale signature of isospin-violating interactions. Although such models have been of great interest in explaining anomalies in data, common theoretically motivated models do not typically exhibit such a cancellation.

The most interesting theoretical scenarios, from the point of view of detectability, are the cases of dark photonmediated interactions $\left(f_{n} / f_{p}=0\right)$ and Z-mediated interactions $\left(f_{p} / f_{n} \sim 0\right)$. These are cases which are closest to the window in which cancellation between proton and neutron response can have a dramatic effect on direct detection sensitivity. We find that, for either the $A^{\prime}$ - or $Z$-mediated scenarios, one can exclude the possibility of isospininvariant interactions at $2 \sigma$ confidence with xenon- and neon-based detectors which each have an exposure about $50 \times$ larger than that required to first obtain evidence for dark matter interactions. If such a model currently just evades searches at XENON1T and PandaX-II, then 100 ton-year exposures of xenon-based and neon-based detectors are sufficient to exclude the possibility of isospin-invariant interactions.

We also considered the case of squark-mediated interactions, but it is unlikely that foreseeable direct detection experiments will have sufficient exposure to distinguish such models from the isospin-invariant scenario.

The most promising experimental setup consists of a high- $Z$ target (such as xenon) and a low- $Z$ target (such as neon). This type of analysis thus requires a neon-based detector with at least 100 ton-year exposure. It will be interesting to study the feasibility of such a detector to exploit an initial discovery of dark matter interactions.

One should note that we have assumed that the dark matter velocity distribution is a nominal Maxwellian distribution, and have not accounted for any uncertainties in the velocity distribution. The extent to which our results are affected if one marginalizes over the velocity distribution is worthy of exploration.

\section{ACKNOWLEDGMENTS}

The work of C. K. is supported in part by Space Florida and the National Aeronautics and Space Administration through the University of Central Florida's NASA Florida
Space Grant Consortium. The work of J. K., D. M. and P. S. is supported in part by NSF CAREER Grant No. PHY1250573, DOE Grant No. DE-SC0010504 and NSF Grant No. PHY-1720282, respectively. D. M. thanks the Aspen Center for Physics (which is supported by NSF Grant No. PHY-1607611) for its hospitality during the completion of this work. C. K., J. K. and P. S. would like to thank CETUP* (Center for Theoretical Underground Physics and Related Areas) for its hospitality and support.

\section{APPENDIX: GERMANIUM- AND ARGON-BASED DETECTORS}

In this appendix, we consider the prospects for distinguishing IVDM from the $f_{n} / f_{p}$ scenario using a xenon-based detector along with either a germaniumbased or argon-based detector. In Fig. 3, we plot exclusion contours of the $f_{n} / f_{p}=1$ scenario, as a function of the true value of $f_{n} / f_{p}$ (left panels) or $f_{p} / f_{n}$ (right panels), and the effective exposure of a future germanium-based direct detection experiment with the same efficiency and recoil energy window as SuperCDMS [38]. In Fig. 4, we present a similar figure in which the effective exposure is for a future argon-based direct detection experiment with the same efficiency and recoil energy window as DarkSide [39]; the $m_{X}=10 \mathrm{GeV}$ case produces a signal below threshold. We have assumed that we also have data from a future xenon-based experiment with a 100 ton-year effective exposure, with the same efficiency and recoil energy window as XENON1T. The exclusion contours of the $f_{n} / f_{p}=1$ hypothesis are at $2 \sigma$ (purple short/long-dashed), $3 \sigma$ (green shortdashed), $4 \sigma$ (black solid) and $5 \sigma$ (blue long-dashed) confidence. In the left column of panels, the vertical grey dashed lines correspond to the values of $f_{n} / f_{p}$ expected for $A^{\prime}$ mediation, $\tilde{u}$ mediation, and $\tilde{d}$ mediation, from left to right. In the right column of panels, the vertical grey dashed lines correspond to the values of $f_{p} / f_{n}$ expected for $Z$ mediation, $\tilde{d}$ mediation, and $\tilde{u}$ mediation, from left to right. In all panels, the solid red contour is the germanium (argon) effective exposure necessary for an expected 2.3 signal events.

As expected, the $f_{n} / f_{p} \sim-0.83$ scenario can be readily distinguished from the isospin-invariant case [36]. However, for either $Z_{2}=$ Ge or Ar, there is always a value of $f_{n} / f_{p}$ that cannot be distinguished from the isospin-invariant case. In fact, this is true for any choice of $Z_{1}$ and $Z_{2}$, simply because the equation $F_{Z_{1}}=F_{Z_{2}}$ is quadratic in $f_{n} / f_{p}$, and thus always has one solution aside from $f_{n} / f_{p}=1$ (unless that solution is degenerate) [36]. Three detectors are required to be able to distinguish an arbitrary value of $f_{n} / f_{p}$ from the isospin-invariant scenario. 

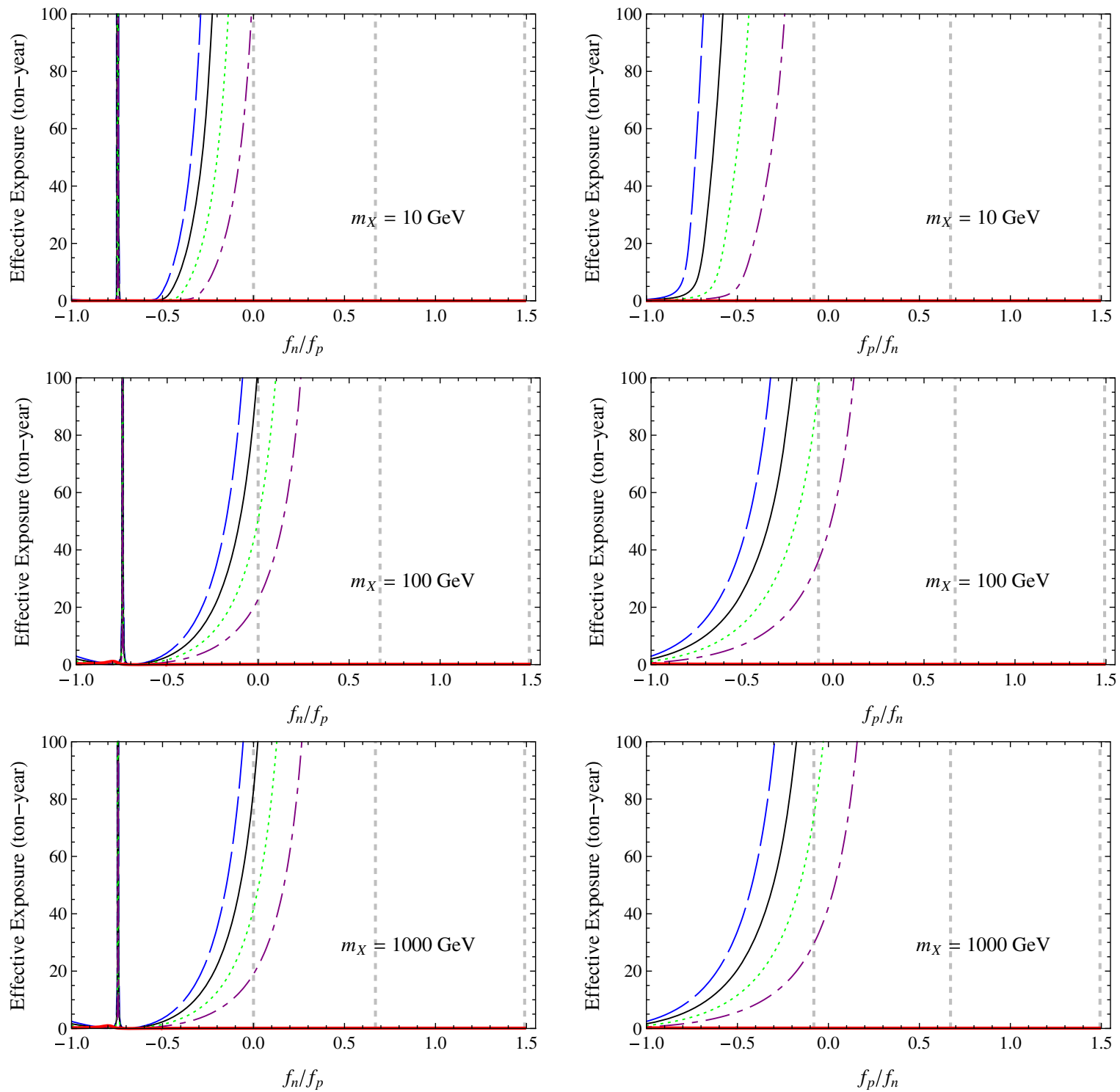

FIG. 3. Exclusion contours of the $f_{n} / f_{p}=1$ scenario, in terms of the true value of $f_{n} / f_{p}$ (left panels) or $f_{p} / f_{n}$ (right panels), and the effective exposure of a germanium-based experiment, assuming a 100 ton-year effective exposure at a xenon-based experiment. The exclusion contours of the $f_{n} / f_{p}=1$ hypothesis are at $2 \sigma$ (purple short/long-dashed), $3 \sigma$ (green short-dashed), $4 \sigma$ (black solid) and $5 \sigma$ (blue long-dashed) confidence. The dashed grey lines correspond, from left to right, to the benchmark cases of $A^{\prime}$ mediation, $\tilde{u}$ mediation, $\tilde{d}$ mediation (left panels), and $Z$ mediation, $\tilde{d}$ mediation, $\tilde{u}$ mediation (right panels). The red line is the effective exposure needed to have 2.3 expected events at a germanium-based experiment. 

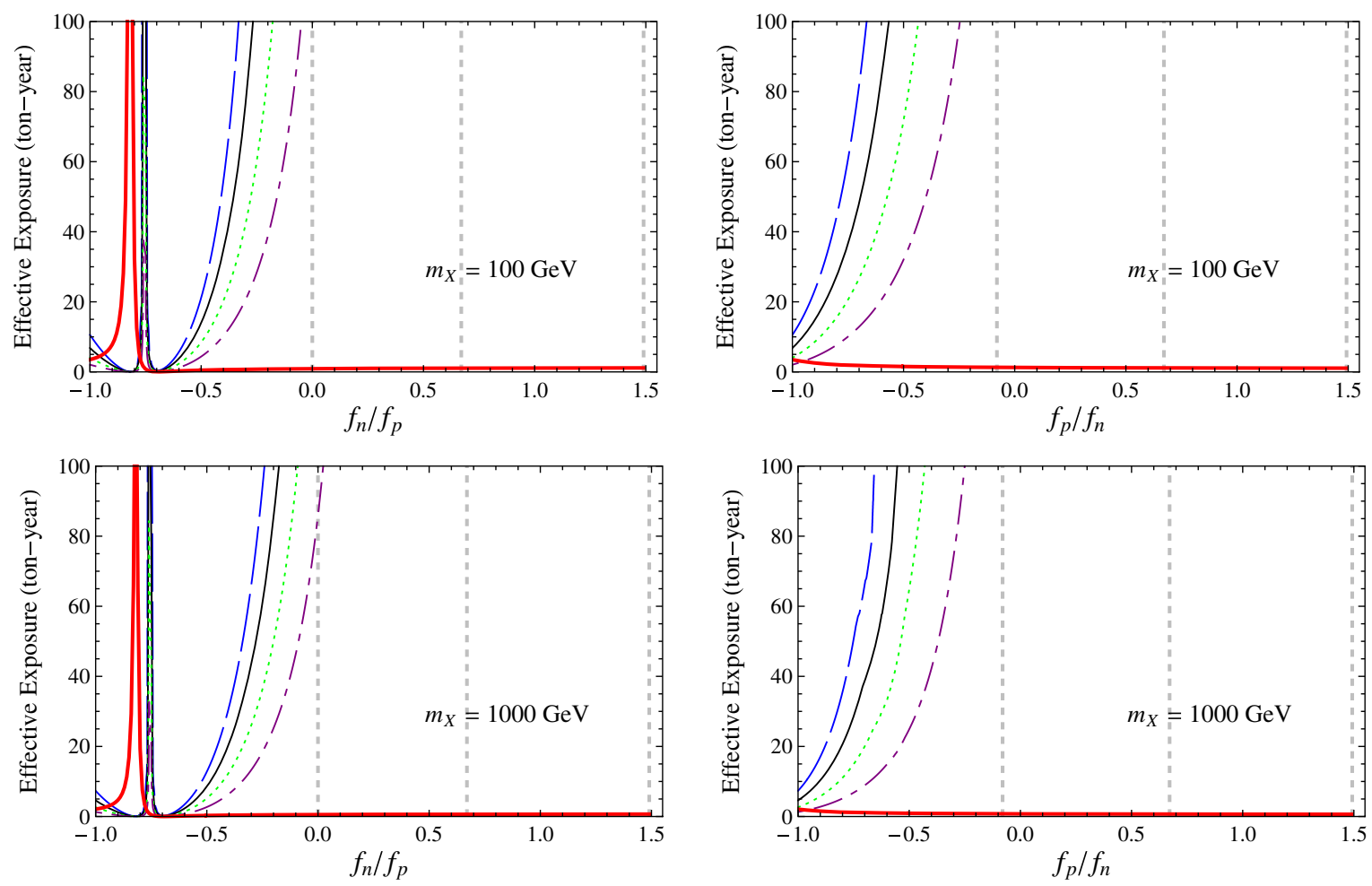

FIG. 4. Similar to Fig. 3, but for an argon-based experiment. The $m_{X}=10 \mathrm{GeV}$ case is absent because the signal is below threshold.

[1] A. Kurylov and M. Kamionkowski, Phys. Rev. D 69, 063503 (2004).

[2] F. Giuliani, Phys. Rev. Lett. 95, 101301 (2005).

[3] S. Chang, J. Liu, A. Pierce, N. Weiner, and I. Yavin, J. Cosmol. Astropart. Phys. 08 (2010) 018.

[4] J. L. Feng, J. Kumar, D. Marfatia, and D. Sanford, Phys. Lett. B 703, 124 (2011).

[5] J. L. Feng, J. Kumar, and D. Sanford, Phys. Rev. D 88, 015021 (2013).

[6] D. S. Akerib et al. (CDMS Collaboration), Phys. Rev. D 82, 122004 (2010).

[7] Z. Ahmed et al. (CDMS-II Collaboration), Phys. Rev. Lett. 106, 131302 (2011).

[8] E. Armengaud et al. (EDELWEISS Collaboration), Phys. Rev. D 86, 051701 (2012).

[9] J. Angle et al. (XENON10 Collaboration), Phys. Rev. Lett. 107, 051301 (2011); Phys. Rev. Lett. 110, 249901(E) (2013).

[10] E. Aprile et al. (XENON100 Collaboration), Phys. Rev. Lett. 107, 131302 (2011).

[11] E. Aprile et al. (XENON100 Collaboration), Phys. Rev. Lett. 109, 181301 (2012).

[12] D. S. Akerib et al. (LUX Collaboration), Phys. Rev. Lett. 118, 021303 (2017).
[13] R. Bernabei et al. (DAMA and LIBRA Collaborations), Eur. Phys. J. C 67, 39 (2010).

[14] C. E. Aalseth et al. (CoGeNT Collaboration), Phys. Rev. Lett. 106, 131301 (2011).

[15] C. E. Aalseth et al. (CoGeNT Collaboration), Phys. Rev. D 88, 012002 (2013).

[16] G. Angloher et al., Eur. Phys. J. C 72, 1971 (2012).

[17] R. Agnese et al. (CDMS Collaboration), Phys. Rev. Lett. 111, 251301 (2013).

[18] X. Gao, Z. Kang, and T. Li, J. Cosmol. Astropart. Phys. 01 (2013) 021.

[19] M. T. Frandsen, F. Kahlhoefer, S. Sarkar, and K. SchmidtHoberg, J. High Energy Phys. 09 (2011) 128.

[20] N. Okada and O. Seto, Phys. Rev. D 88, 063506 (2013).

[21] G. Blanger, A. Goudelis, J. C. Park, and A. Pukhov, J. Cosmol. Astropart. Phys. 02 (2014) 020.

[22] K. Hamaguchi, S. P. Liew, T. Moroi, and Y. Yamamoto, J. High Energy Phys. 05 (2014) 086.

[23] V. M. Lozano, M. Peir, and P. Soler, J. High Energy Phys. 04 (2015) 175.

[24] A. Drozd, B. Grzadkowski, J. F. Gunion, and Y. Jiang, J. Cosmol. Astropart. Phys. 10 (2016) 040.

[25] E. Aprile et al. (XENON Collaboration), Phys. Rev. Lett. 119, 181301 (2017). 
[26] X. Cui et al. (PandaX-II Collaboration), Phys. Rev. Lett. 119, 181302 (2017).

[27] J. Kumar and D. Marfatia, Phys. Rev. D 88, 014035 (2013).

[28] L. B. Okun, Zh. Eksp. Teor. Fiz. 83, 892 (1982) [Sov. Phys. JETP 56, 502 (1982)].

[29] B. Holdom, Phys. Lett. 166B, 196 (1986).

[30] C. Boehm and P. Fayet, Nucl. Phys. B683, 219 (2004).

[31] M. Pospelov, Phys. Rev. D 80, 095002 (2009).

[32] M. Drees and M. Nojiri, Phys. Rev. D 48, 3483 (1993).

[33] K. R. Dienes, C. F. Kolda, and J. March-Russell, Nucl. Phys. B492, 104 (1997).
[34] C. Kelso, J. Kumar, P. Sandick, and P. Stengel, Phys. Rev. D 91, 055028 (2015).

[35] A. Davidson, C. Kelso, J. Kumar, P. Sandick, and P. Stengel, Phys. Rev. D 96, 115029 (2017).

[36] J. L. Feng, J. Kumar, D. Marfatia, and D. Sanford, arXiv: 1307.1758.

[37] D. N. McKinsey and K. J. Coakley, Astropart. Phys. 22, 355 (2005).

[38] R. Agnese et al. (SuperCDMS Collaboration), Phys. Rev. D 95, 082002 (2017).

[39] C. E. Aalseth et al., arXiv:1707.08145. 